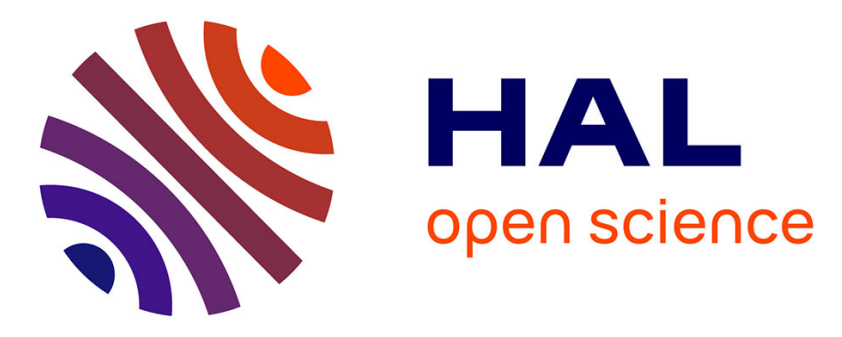

\title{
Resolution Enhancement in Multidimensional Solid-State NMR Spectroscopy of Proteins using Spin-State Selection
}

\author{
Luminita Duma, Sabine Hediger, Bernhard Brutscher, Anja Böckmann, \\ Lyndon Emsley
}

\section{To cite this version:}

Luminita Duma, Sabine Hediger, Bernhard Brutscher, Anja Böckmann, Lyndon Emsley. Resolution Enhancement in Multidimensional Solid-State NMR Spectroscopy of Proteins using Spin-State Selection. Journal of the American Chemical Society, 2003, 125, pp.11816. 10.1021/ja036893n . hal-00078170

\section{HAL Id: hal-00078170 \\ https://hal.science/hal-00078170}

Submitted on 5 Jun 2006

HAL is a multi-disciplinary open access archive for the deposit and dissemination of scientific research documents, whether they are published or not. The documents may come from teaching and research institutions in France or abroad, or from public or private research centers.
L'archive ouverte pluridisciplinaire HAL, est destinée au dépôt et à la diffusion de documents scientifiques de niveau recherche, publiés ou non, émanant des établissements d'enseignement et de recherche français ou étrangers, des laboratoires publics ou privés. 


\title{
Resolution Enhancement in Multidimensional Solid-State NMR of ${ }^{13} \mathrm{C}$-Labeled Proteins Using Spin-State Selection
}

\author{
Luminita Duma $^{(\mathrm{a})}$, Sabine Hediger ${ }^{\star(a)}$, Bernhard Brutscher ${ }^{(\mathrm{b})}$, Anja Böckmann $^{(\mathrm{c})}$, Lyndon Emsley ${ }^{\star(a)}$ \\ ${ }^{(a)}$ Laboratoire de Chimie, UMR 5532 CNRS/ENS, Ecole Normale Supérieure de Lyon, 69364 Lyon, France, ${ }^{(b)}$ Institut \\ de Biologie Structurale Jean-Pierre Ebel CNRS/CEA, 38027 Grenoble, France, and ${ }^{(c)}$ Institut de Biologie et Chimie \\ des Protéines, UMR 5086 CNRS, 69367 Lyon, France. \\ RECEIVED DATE; *Email: Sabine.Hediger@ens-Iyon.fr, Lyndon.Emsley@ens-lyon.fr
}

Much progress has recently been made in the field of solidstate NMR of isotope $\left({ }^{13} \mathrm{C},{ }^{15} \mathrm{~N}\right)$ enriched bio-molecules, leading to the first protein structure solved by solid-state NMR. ${ }^{1}$ One of the principal factors limiting the study of larger systems remains spectral resolution. In uniformly ${ }^{13} \mathrm{C}$-labeled compounds such as proteins, the ${ }^{13} \mathrm{C}-{ }^{13} \mathrm{C} \quad J$-couplings constitute a significant contribution to the linewidth in magic-angle-spinning (MAS) spectra. $J$-decoupling techniques for solid-state NMR using semiselective pulses were first proposed in 1996 by Straus et al. ${ }^{2}$ to enhance the spectral resolution in indirectly detected spectral dimensions. Here, we present the application of spin-state selection and transition-selective polarization transfer to multidimensional solid-state NMR correlation experiments of ${ }^{13} \mathrm{C}$ labeled proteins. We show that single-transition selection removes the line broadening due to the $J_{C O C \alpha}$ spin coupling in both direct and indirect dimensions of a two-dimensional $\mathrm{CO}-\mathrm{C}^{\alpha}$ correlation experiment. The experiment is demonstrated on a sample of microcrystalline $\mathrm{Crh}$, a 85 -residue protein involved in carbon catabolite repression in Bacillus subtilis ${ }^{3}$ and leads to nearly a factor two improvement in spectral resolution.

Spin-state-selective NMR techniques have been developed in liquid-state NMR spectroscopy for the measurement of small spin-spin coupling constants ${ }^{4}$ and for transverse-relaxationoptimized spectroscopy (TROSY). ${ }^{5}$ Recently, we have demonstrated the feasibility of homonuclear spin-state selection in the solid state, ${ }^{6}$ even for $J$-couplings that are not resolved, using an IPAP-type selection filter. ${ }^{4}$ In order to perform a multidimensional experiment, the selected transition of the first spin, $I$, evolving during $t_{1}$ should be transferred to a single-transition of a second spin, $S$, by means of an appropriate mixing sequence. Generally, spin-state-selective coherence transfer is obtained through zero-quantum (ZQ) or double-quantum (DQ) rotations. ${ }^{7}$ A ZQ rotation "conserves" the spin state $\left(I_{x} S^{\alpha} \rightarrow I^{\alpha} S_{x}\right)$ and a DQ rotation "reverses" the spin state $\left(I_{x} S^{\alpha} \rightarrow I^{\beta} S_{x}\right)$. In liquid-state NMR, spin-state-selective coherence transfer is obtained using planar mixing or $\mathrm{S}^{3} \mathrm{CT}$ building blocks. ${ }^{8}$ Similar $J$-based spinstate-selective transfer techniques could be envisaged in the solid state. However, schemes using the dipolar coupling may be more appropriate for solid-state applications since most of the (many) existing solid-state dipolar correlation techniques under MAS have a ZQ or DQ average Hamiltonian, and may therefore be used directly for spin-state-selective polarization or coherence transfer. Common examples of such sequences are proton-driven spin diffusion (PDSD) ${ }^{9}$ and RFDR, ${ }^{10}$ with a ZQ-average Hamiltonian, and C7, POST-C7, and SPC5 with a DQ-average Hamiltonian. ${ }^{11}$

The pulse sequence shown in Figure 1 yields a spin-stateselective $\mathrm{CO}-\mathrm{C}^{\alpha}$ correlation experiment using PDSD. After crosspolarization, both $I(\mathrm{CO})$ transitions are separated into different sub-spectra with respect to the $S\left(\mathrm{C}^{\alpha}\right)$ spin by an IPAP sequence.
The $t_{1}$ period is built into the IPAP sequence providing a constant time (CT) experiment. ${ }^{12}$ PDSD then yields spin-state-selective $\mathrm{CO}->\mathrm{C}^{\alpha}$ polarization transfer. During the PDSD mixing period, $\mathrm{CO}$ polarization $\left(I_{z}\right)$ is transferred to $\mathrm{C}^{\alpha}$ polarization $\left(S_{z}\right)$ by the dipolar coupling, but the two-spin order $2 I_{z} S_{z}$, present after $t_{1}$ evolution, is not affected by the dipolar coupling. Since the buildup of $S_{z}$ and the decay of $2 I_{z} S_{z}$ are dependent on the mixing time $\tau_{\text {mix }}$, and in order to obtain proper spin-state selection independent of $\tau_{\text {mix }}$, the two polarization-transfer pathways are separated into different sub-spectra by an appropriate 2-step phase cycle, as detailed in the caption of Figure 1. By separating the in-phase and anti-phase components of the $S$-spin coherence during detection $\left(t_{2}\right)$, their relative amplitude can be adjusted by an appropriate scaling factor $k$, to yield proper spin-state selection.

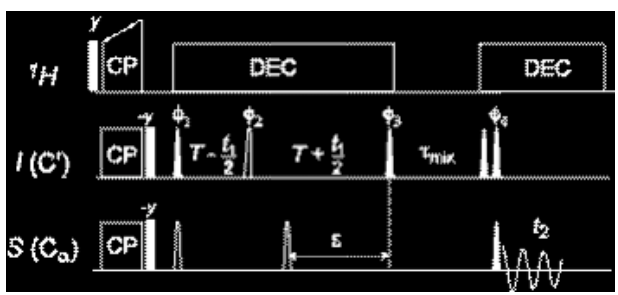

Figure 1. Pulse sequence suitable for an $I-S$ (e.g. CO-C ${ }^{\alpha}$ ) correlation experiment. $90^{\circ}$ and $180^{\circ}$ rf pulses are represented by filled and open bars, respectively. The semi-selective $\mathrm{CO}$ pulses are applied with the shape of the center lobe of a $\sin x / x$ function, whereas the $\mathrm{C}_{\alpha}$ pulses were rectangular with $\omega_{1}=\Delta / \operatorname{sqrt}(15)\left(90^{\circ}\right)$ and $\Delta / \operatorname{sqrt}(3)\left(180^{\circ}\right)$. All pulses are applied along the $x$ axis unless indicated. An 8-step phase cycle was applied with $\phi_{1}=x^{4}-x^{4} ; \phi_{2}=x y-x-y ; \phi_{\mathrm{rec}}=x-x \quad x-x-x \quad x-x \quad x$. The constant time delay $2 T$ is adjusted to $\left(2 J_{\mathrm{CC} \alpha}\right)^{-1}$. Four data sets (A1), (A2), (B1) and (B2) are recorded. A- and B-type experiments are used to separate the $I$-spin transitions using IPAP with the following settings: (A) $\varepsilon=T, \phi_{3}=-x$ and (B) $\varepsilon=0, \phi_{3}=y$. Each of the experiments A and B are recorded twice by setting alternatively the phase $\phi_{4}$ to $x$ and $-x$. Addition of the two data sets yields in-phase selection (A1, B1) of the $S$-spin coherence, subtraction yields anti-phase selection (A2, B2). Linear combination $(\mathrm{A} 1+\delta \times \mathrm{B} 1) \pm k \times(\mathrm{A} 2+\delta \times \mathrm{B} 2)$, yields the four different single-transition correlation spectra $(\alpha \alpha, \alpha \beta, \beta \alpha, \beta \beta)$ with $\delta=+1$ or -1 and $k$ a scaling factor taking into account the different PDSD transfer functions described in the text. The pulse sequence and phase cycle used here are available from our website, ${ }^{13}$ or upon request to the authors.

Figure 2 shows the $(\alpha \alpha)$-sub-spectrum recorded for a microcrystalline sample of uniformly ${ }^{13} \mathrm{C}-{ }^{15} \mathrm{~N}$ labeled $\mathrm{Crh}$ using the sequence of Figure 1, compared to a standard PDSD correlation spectrum. Spin-state selection provides a remarkable increase in resolution in both dimensions. Specifically, the resolution of the G49 cross peak is enhanced by $44 \%$ and $17 \%$ for $\mathrm{C}^{\alpha}$ and $\mathrm{CO}$ dimensions, respectively. The resolution enhancement is more pronounced in the $\mathrm{C}^{\alpha}$ dimension because of the longer acquisition time used for direct detection. We have limited here 
the spectral resolution in the $\mathrm{CO}$ dimension to the maximal CT of $9.2 \mathrm{~ms}$. It can be further increased by lengthening $t_{1}$ in an additional non-constant acquisition time or by processing using stronger apodization functions and linear prediction (LP). Despite the CT frequency encoding, advanced prediction algorithms like mirror-image LP can not be used here. Indeed, in solid-state $\mathrm{NMR}$, the ${ }^{13} \mathrm{C}$ linewidth is dominated by refocusing interactions $\mathrm{s}^{14}$ and the signal therefore still decays during the CT evolution period.

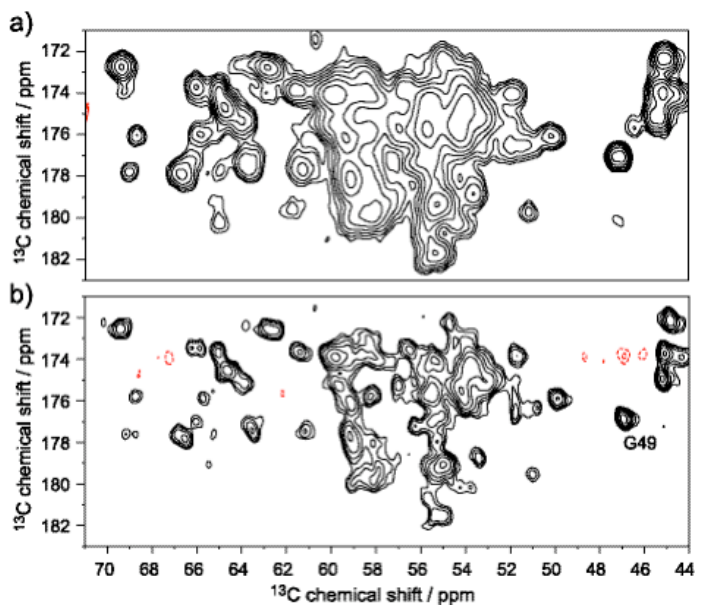

Figure 2. $\mathrm{CO}-\mathrm{C}^{\alpha}$ region of the standard (a) and the $(\alpha \alpha)$-spin-state selective (b) PDSD spectrum of microcrystalline Crh. The second spectrum was obtained from the linear combination $\mathrm{A} 1+\mathrm{B} 1+k \times(\mathrm{A} 2+$ B2), with $k=0.7$. Both experiments were performed on a Bruker Avance spectrometer operating at a ${ }^{1} \mathrm{H}$ frequency of $500.13 \mathrm{MHz}$ with a $4 \mathrm{~mm}$ double-resonance CP/MAS probe. The temperature was set to $269 \mathrm{~K}$, and the MAS frequency was $11 \mathrm{kHz}$. SPINAL ${ }^{15}{ }^{1} \mathrm{H}$ decoupling was used with $\omega_{1}=78 \mathrm{kHz}$. $\tau_{\text {mix }}$ was $30 \mathrm{~ms}$, and $t_{2}{ }^{\max }$ was $25 \mathrm{~ms}$. For the standard and the spin-state-selective spectra, a total of 64 and $4 \times 24$ transients respectively were accumulated for each of 240 complex points in $t_{1}$. Cosine apodization was applied in both dimensions prior to Fourier transformation. For both spectra, the first contour level was set to $15 \%$ of the intensity of the G49 resonance, with a factor of 1.4 between levels.

The efficiency of the spin-state-selective experiment compared to the standard PDSD experiment was estimated from measured cross peak intensities to be about $35 \%$. This loss is mainly due to the $2 T \approx 9 \mathrm{~ms}$ IPAP filter. As we have shown recently, ${ }^{6}$ this signal loss is greatly reduced by using high-power ${ }^{1} \mathrm{H}$ decoupling during the filter delay and faster spinning of the sample. This is possible for protein samples if a better cooling system than we have is available. In addition, the four sub-spectra can be added after appropriate shifting of the spectrum by $\pm J_{C O C \alpha} / 2 \approx 27 \mathrm{~Hz}$ along the $\mathrm{CO}$ and/or $\mathrm{C}^{\alpha}$ dimensions, resulting in an additional gain of a factor of two in signal to noise. Therefore we expect that for an optimized experimental setup the spin-state-selective experiment not only increases spectral resolution but also sensitivity.

Figure 3 illustrates how the recorded 2D data sets are combined. The linear combinations $(\mathrm{A} 1+\delta \times \mathrm{B} 1) \pm \mathrm{k} \times(\mathrm{A} 2+$ $\delta \times B 2)$ yield all four single-transition-to-single-transition correlation spectra $(\alpha \alpha),(\alpha \beta),(\beta \alpha)$, and $(\beta \beta)$. For $\tau_{\text {mix }}=30 \mathrm{~ms}$, the scaling factor was found to be $k=0.7$. For spectra with $\tau_{\text {mix }}=$ $15 \mathrm{~ms}$, a scaling factor $k=0.5$ was found.

At longer mixing times some polarization is transferred by PDSD from the $\mathrm{CO}$ to other side chain carbons $\left(C^{\beta}, C^{\gamma}, C^{\delta}\right)$. Since there is no direct scalar coupling between the $\mathrm{CO}$ and the side chain carbons, there is no frequency shift along the detection dimension $\left(\omega_{2}\right)$ between the $(\alpha \alpha)$ and $(\alpha \beta)$, or $(\beta \alpha)$ and $(\beta \beta)$ subspectra. Thus the new experiment provides a simple way of distinguishing $\mathrm{C}^{\alpha}$ from other side chain carbons (e.g. $\mathrm{C}^{\beta}$ of $\mathrm{Thr}$ and Ser residues) by comparison of sub-spectra.

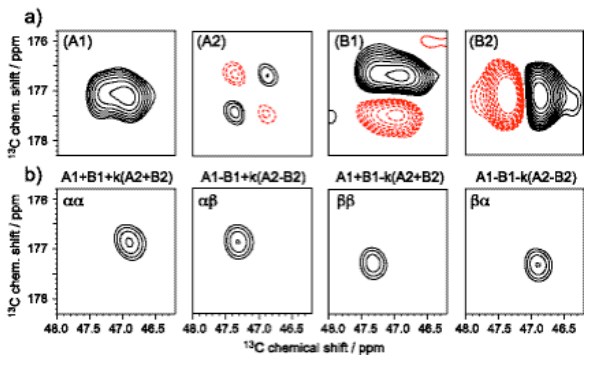

Figure 3. a) Sub-spectra (A1), (A2), (B1), and (B2) recorded using the spin-state-selective CO-C ${ }^{\alpha}$-PDSD experiment of Figure 1. Experimental details are given in the caption of Figure 2. b) Separation of the four crosspeak transitions obtained by linear combination of the spectra in (a). For clarity, only the G49 cross peak region is shown. Contours are drawn at the same levels for all spectra.

In conclusion, we have introduced a new experiment providing significant resolution enhancement in multi-dimensional solidstate NMR correlation experiments. Resolution enhancement is achieved by using transition-selective excitation and transfer techniques. Spin-state-selective polarization transfer is obtained using standard ZQ solid-state NMR mixing sequences. Similar results are expected for transfer sequences based on DQ rotations. The new experiment can be easily extended to higher-dimensional experiments. In addition, spin-state-selective correlation experiments allow the distinction of 'direct' transfer peaks, involving covalently-bound nuclei, and 'relayed' transfer peaks. The new experiment is expected to be very useful for the assignment of solid-state NMR spectra of proteins.

(1) Castellani, F.; van Rossum, B.; Diehl, A.; Schubert, M.; Rehbein, K.; Oschkinat, H. Nature 2002, 420, 98-102.

(2) Straus, S. K.; Bremi, T.; Ernst, R. R. Chem. Phys. Lett. 1996, 262, 709715 .

(3) (a) Favier, A.; Brutscher, B.; Blackledge, M.; Galinier, A.; Deutscher, J.; Penin, F.; Marion, D. J. Mol. Biol. 2002, 317, 131-144. (b) Böckmann, A.; Lange, A.; Galinier, A.; Luca, S.; Giraud, N.; Juy, M.; Heise, H.; Montserret, R.; Penin, F.; Baldus, M. J. Biomol. NMR 2003, (accepted).

(4) (a) Ottiger, M.; Delaglio, F.; Marquardt, J. L.; Tjandra, N.; Bax, A. J. Magn. Reson. 1998, 134, 365-369. (b) Andersson, P.; Weigelt, J.; Otting, G. J. Biomol. NMR 1998, 12, 435-441.

(5) Pervushin, K.; Riek, R.; Wider, G.; Wüthrich, K. Proc. Natl. Acad. Sci. U. S. A. 1997, 94, 12366-12371.

(6) Duma, L.; Hediger, S.; Lesage, A.; Emsley, L., J. Magn. Reson. 2003 (in press).

(7) Meissner, A.; Duus, J. O.; Sørensen, O. W. J. Biomol. NMR 1997, 10, 8994.

(8) Sørensen, M. D.; Meissner, A.; Sørensen, O. W. J. Biomol. NMR 1997, $10,181-186$

(9) Bloembergen, N. Physica 1949, 15, 386-426

(10) Bennett, A. E.; Oak, J. H.; Griffin, R. G.; Vega, S. J. Chem. Phys. 1992 96, 8624-8627.

(11) (a) Lee, Y. K.; Kurur, N. D.; Helmle, M.; Johannessen, O. G.; Nielsen, N. C.; Levitt, M. H. Chem. Phys. Lett. 1995, 242, 304-309. (b) Hohwy, M.; Jakobsen, H. J.; Eden, M.; Levitt, M. H.; Nielsen, N. C. J. Chem. Phys. 1998, 108, 2686-2694. (c) Hohwy, M.; Rienstra, C. M.; Jaroniec, C. P. Griffin, R. G. J. Chem. Phys. 1999, 110, 7983-7992.

(12) Bax, A.; Mehlkopf, A. F.; Smidt, J. J. Magn. Reson. 1979, 35, 167-169.

(13) http://www.ens-lyon.fr/STIM/NMR

(14) Lesage, A.; Bardet, M.; Emlsey, L. J. Am. Chem. Soc. 1999, 121, $10987-$ 10993.

(15) Fung, B. M.; Khitrin, A. K.; Ermolaev, K. J. Magn. Reson. 2000, 142 , 97-101. 